\title{
Identification of Postoperative Margins of Glioblastoma Multiforme Using Gold Nanoparticles Conjugated to Epidermal Growth Factor Antibodies
}

\begin{abstract}
Background: Glioblastoma multiforme (GBM) is the most common primary malignant brain tumor in adults. Complete resection is impossible. Overexpression of epidermal growth factor receptor (EGFR) by the tumor has been associated with the level of malignancy and possibly with prognosis. Monoclonal antibodies may be used to identify tumor borders. The aim of the present study was to investigate the value of immunostaining with anti-EGFR antibodies conjugated to gold nanoparticles (GNPs) for detecting tumor infiltration into adjacent tissue.
\end{abstract}

Methods: Fresh GBM samples were collected intraoperatively from 14 patients treated at a tertiary medical center in 2014-2015, in accordance with the guidelines of the local and national ethic committees. Demographic, clinical, and outcome data of the patients were recorded. Analysis for EGFR overexpression by polysomy or amplification was determined by calculating the ratio of expression between pairs of the following genes: EGFR, GPER, and RNaseP. Tumor sample with known overexpression of EGFR underwent immunostaining with GNPconjugated anti-EGFR antibodies followed by light-scattering spectroscopy and electron microscopy. The findings were compared with routine hematoxylin and eosin staining.

Results: On molecular analysis, EFGR overexpression was identified in 10/14 GBM samples (71\%). Amplification was detected in $50 \%$ and polysomy in $27 \%$, and one sample showed both. Patients whose samples were characterized by an overexpression of EGFR died within 3-10 months, whereas 2 of the patients with normal EGFR expression were still alive 9 and $<15$ months after surgery. Tumor borders identified by anti-EGFR-GNP immunostaining exceeded the area considered "healthy" on routine pathologic study.

Conclusion: The high level of expression of EGFR in the majority of GBM tumors makes it possible to selectively distinguish tumor infiltration using anti-EGFR antibodies conjugated to GNPs. As staining with GNPs is tumor-functionaldependent, the findings may have important prognostic value. Preoperative mapping of tumor extent may facilitate complete resection of these difficult-toresect tumors.

Keywords: GBM; Glioblastoma multiforme; Gold nanoparticles; GNPs; Tumor margins; EGFR; Epidermal growth factor; Overexpression

Abbreviations: GBM: Glioblastoma Multiforme; GNP: Gold Nanoparticles; H\&E: Hematoxylin and Eosin; EGFR: Epidermal Growth Factor Receptor

Received: October 23, 2016; Accepted: November 25, 2016; Published: November 28, 2016

\author{
David Hazon "1", \\ Neelan J Marianayagam 1 , \\ Orit Barinfeld², \\ Shalom Michowiz ${ }^{1}$, Susana \\ Fichman-Horn ${ }^{3}$, \\ Asaf Olshinka ${ }^{4}$, \\ Ella Kaganovsky ${ }^{3}$, \\ Avraham Hirshberg ${ }^{5}$, \\ Dror Fixler 6 and \\ Nitza Goldenberg-Cohen ${ }^{4,7,8 \#}$
}

1 Departments of Neurosurgery, Rabin Medical Center, Beilinson Hospital, Petach Tikva, Israel

2 The Krieger Eye Research Laboratory, Felsenstein Medical Research Center, Petach Tikva, Israel

3 Department of Pathology, Rabin Medical Center, Beilinson Hospital, Petach Tikva, Israel

4 Department of Plastic Surgery, Rabin Medical Center, Beilinson Hospital, Petach Tikva, Israel

5 Department of Oral Pathology and Oral Medicine, Maurice and Gabriela Goldschleger School of Dental Medicine, Tel Aviv University, Israel

6 Faculty of Engineering and Institute of Nanotechnology and Advanced Materials, Bar Ilan University, Ramat Gan, Israel

7 Department of Ophthalmology, Bnai Zion Medical Center, Haifa, Israel

8 Sackler Faculty of Medicine, Tel Aviv University, Tel Aviv, Israel

\# These authors contributed equally to this manuscript.

Corresponding author: Nitza Goldenberg-Cohen

झ ncohen1@gmail.com

MD, Chair, Ophthalmology Department, Bnai Zion Medical Center, Haifa 3339419, Israel. 


\section{Background}

The epidermal growth factor (EGFR) gene (7p12.1) codes a cell membrane protein receptor with intrinsic tyrosine kinase activity. It has been the subject of rigorous investigation owing to its involvement in several human cancers and as a potential target of therapy [1]. The EGFR protein is activated by ligand binding which leads to tightly regulated stimulation of proliferative and survival cell-signaling pathways, most notably the RAS/RAF/MEK/ MAPK and PI3K/Akt [2-4].

Glioblastoma Multiforme (GBM) is the most common primary brain tumor in adults. Despite current therapies, it has a rapidly progressive and fatal course [5]. A recent study of the molecular genetics of GBM reported overexpression of the EGFR gene in the majority of tumors evaluated, either by amplification, which results in multiple copies of the gene in chromosomal regions (amplicons) [2] or by polysomy of chromosome 7 which contains the EGFR gene [6]. In very rare cases, EGFR overexpression may be due to a de novo mutation [4] As a result of these findings, attention has been addressed to overexpression of the EGFR protein as a potential prognostic indicator in GBM [5]. However, its accuracy remains unclear $[3,5,7,8]$.

Complete resection of GBM with clear margins is nearly impossible owing to its aggressive nature and diffuse structure $[9,10]$. GBM has a high recurrence rate after surgery even when combined with chemotherapy or radiotherapy $[9,11]$. The estimated oneyear mortality rate is as high as $65 \%$ [10]. The diffuse structure of the tumor can also hinder intraoperative and post-resection mapping of the tumor margins by standard imaging techniques. Therefore, a better method for mapping tumor margins would be invaluable to neurosurgeons and could contribute to patient survival.

The application of biocompatible gold nanoparticles (GNPs) for in vivo molecular imaging has attracted much interest in various scientific disciplines, such as biomedicine, engineering, and chemistry [12]. Recently, our group suggested a new method for cancer detection based on optical measurements of GNPs [13]. The GNPs were bioconjugated to EGFR-expressing head and neck cancer cells. The accumulation of the GNPs in the cancer cells caused an increase in their absorption properties, resulting in a specific reflection signal of the tumor site $[14,15]$.

Prompted by these findings, in the present study, we sought to determine if GNP-conjugated anti-EGFR antibodies can be used to mark tumor borders and to identify tumor infiltration into surrounding tissue.

\section{Methods}

\section{Ethics, consent and permissions}

The study was approved by the national and institutional ethics committees.

\section{Glioblastoma tissue samples}

The study was conducted on fresh GBM tumor samples from 14 consecutive patients treated at Rabin Medical Center in 2014-
Fax: $+972-3-9211478$

Citation: Hazon D, Marianayagam NJ, Barinfeld O, et al. Identification of Postoperative Margins of Glioblastoma Multiforme Using Gold Nanoparticles Conjugated to Epidermal Growth Factor Antibodies. Nano Res Appl. 2016, 2:2.

2015. The diagnosis of GBM was made clinically, radiologically, and pathologically. All participants gave written informed consent prior to surgery, and the samples were collected intraoperatively in accordance with the guidelines of the ethics committees. The tissue diagnosis was confirmed by a pathologist (S.H.F.) using the glioma grading scale of the World Health Organization [16].

\section{Patient data}

The demographic and clinical data of the patients were recorded, and the clinical course was compared with the molecular data and outcome.

\section{Molecular analysis}

DNA extraction from tumor tissue. The fresh tumor samples were immediately placed in Proteinase $\mathrm{K}$ for 12 hours at $56^{\circ} \mathrm{C}$ for digestion of all cellular components except DNA. DNA was extracted using the Blood and Tissue DNAeasy kit (Qiagen, Hilden, Germany) according to the manufacturer's protocol. All DNA obtained from the tumor samples was stored at $-20^{\circ} \mathrm{C}$ for further analysis.

\section{Testing EGFR expression levels in GBM samples}

To determine EGFR expression in the GBM samples, we measured the expression of the GPER and RNaseP genes, as recently described [6]. The primer sequence was kindly provided by $\mathrm{Dr}$. Bieńkowski and his group. The following sense and antisense primers were used:

EGFR: 5'-CACACCCCTGACTCTCCACT-3' and 5'-GAGACAATCCTGTGAGCTTGG-3';

GPER: 5'-CATCTGGACGGCAGGTAAGT-3' and 5'-CCCTCAGCCGGTAGTTTTC-3';

RNaseP: 5'-GGGAGATGCGGAAGAATGT-3' and 5'-CCTCCAGTCAGCCACAGAA-3'.

GPER lies in close proximity to EGFR on chromosome 7; RNaseP is a housekeeping gene on chromosome 15. A ratio of GPER expression to RNaseP expression above 1.5 is considered indicative of chromosome 7 polysomy. A ratio of EGFR expression to $R$ Nase $P$ expression above 1.5 is considered indicative of $E G F R$ amplification [6]. Cut off was calculated according to a previous publication [6].

\section{Real-time-polymerase chain reaction (RT-PCR)}

RT-PCR was performed with the SYBR ${ }^{\circ}$ Fast PCR system (Applied Biosystems). DNA samples were loaded with the appropriate primers and reagents to a final volume of $10 \mu \mathrm{l}$, with a final concentration of $10 \mathrm{ng}$ DNA. The following materials were added to the reaction mixture: Sybr Green $(5 \mu \mathrm{l}), 10 \mu \mathrm{M}$ reverse primer $(0.5 \mu \mathrm{l}), 10 \mu \mathrm{M}$ forward primer $(0.5 \mu \mathrm{l})$, DNA $(10 \mu \mathrm{l})$ and $\mathrm{ddH}_{2} \mathrm{O}(3$ 
$\mu$ ). The thermal cycling conditions for RT-PCR were as follows: enzyme activation, 19 minutes at $95^{\circ} \mathrm{C}$; denaturation, 40 cycles of 15 seconds at $95^{\circ} \mathrm{C}$; annealing and extension, 1 minute at $60^{\circ} \mathrm{C}$. Melting curves were generated to analyze the specificity of gene amplification. The expression levels of each gene of interest were calculated from the generated data $[6,9,16]$.

\section{Immunostaining}

Preparation of GNPs and their conjugation to anti-EGFR antibody. Rod-shaped GNPs were used, with a spectral peak of $660 \mathrm{~nm}[17,18]$. The GNPs were conjugated to two different anti-EGFR antibodies: a recombinant human/mouse chimeric EGFR monoclonal antibody [Cetuximab (Erbitux), Merck KGaA, Germany] and an affinity purified rabbit polyclonal antibody (PDGFR- $\alpha$, Santa Cruz Biotechnology, Santa Cruz, CA, USA). The GNPs were bioconjugated to the anti-EGFR antibodies using polystyrene sulfonate, according to the method described by Lvov [19].

\section{Immunostaining with ant-EGFR conjugated to GNPs}

Paraffin-embedded block from each patient containing tumor tissue were collected from the pathology department. Two 4 $\mu \mathrm{m}$ sections were cut on glass slides. One slide was stained with hematoxylin and eosin ( $\mathrm{H} \& \mathrm{E})$ and evaluated by a pathologist for tumor borders. The other was de-paraffinized, marked with the tumor borders identified on the H\&E-stained slide, and stained with anti-EGFR antibody conjugated to GNPs, as previously described $[13,14]$.

\section{Hyperspectral imaging of immunostained slides}

The reflectance of GNPs within the tissue samples was measured with the hyperspectral imaging system (Nuance, CRi, Woburn, MA, USA). For imaging in RGB mode, we used a 40x objective (0.75 NA) 32-bit ultrasensitive charge-coupled device camera detector (N-MSI-EX) under halogen illumination (UN2-PSE100, Nikon, Tokyo, Japan) Microscopy was performed with a Nikon 80i microscope. Images were acquired using Nuance 2.1 software (Burlington, MA, USA). The reflectance is presented as arbitrary intensity units (IU).

Each slide was scanned before (negative control) and after GNPs where added. Under both conditions, the reflectance spectra were measured in 3 areas on the tumor and 3 areas on the area considered healthy by routine pathology. For image processing analysis, the results without GNPs were subtracted from the results with GNPs. We subtracted the spectra of the pure glass slide in order to reduce noise.

\section{Electron microscopy of immunostained slides}

To directly visualize GNPs in the tissue, we scanned the GNPstained slides with the airSEM ${ }^{\mathrm{TM}}$ (B-Nano, Krapina, Croatia), an innovative high- resolution scanning electron microscope that sidesteps the main obstacles stemming from the need for vacuum as a prerequisite for capture of high-resolution images. The technology overcomes fundamental electron beam imaging-related challenges, such as scattering of electrons by gas molecules and efficient signal collection, leading to a resolution better than $5 \mathrm{~nm}$ under ambient conditions.

\section{Results}

The patient data are summarized in Table 1. A sample of GBM neuroimaging is shown in Figure 1.

\section{EGFR overexpression in GBM}

EGFR overexpression was observed in 10 of the 14 (71\%) GBM samples examined (Table 1). Amplification was found in 5 tumor samples, polysomy in 4 samples, and the both amplification and polysomy in one sample. The remaining 4 samples showed normal EGFR expression patterns.

Table 1 Characteristics of patients with GBM: Demographics and EGFR expression status and suggested mechanism.

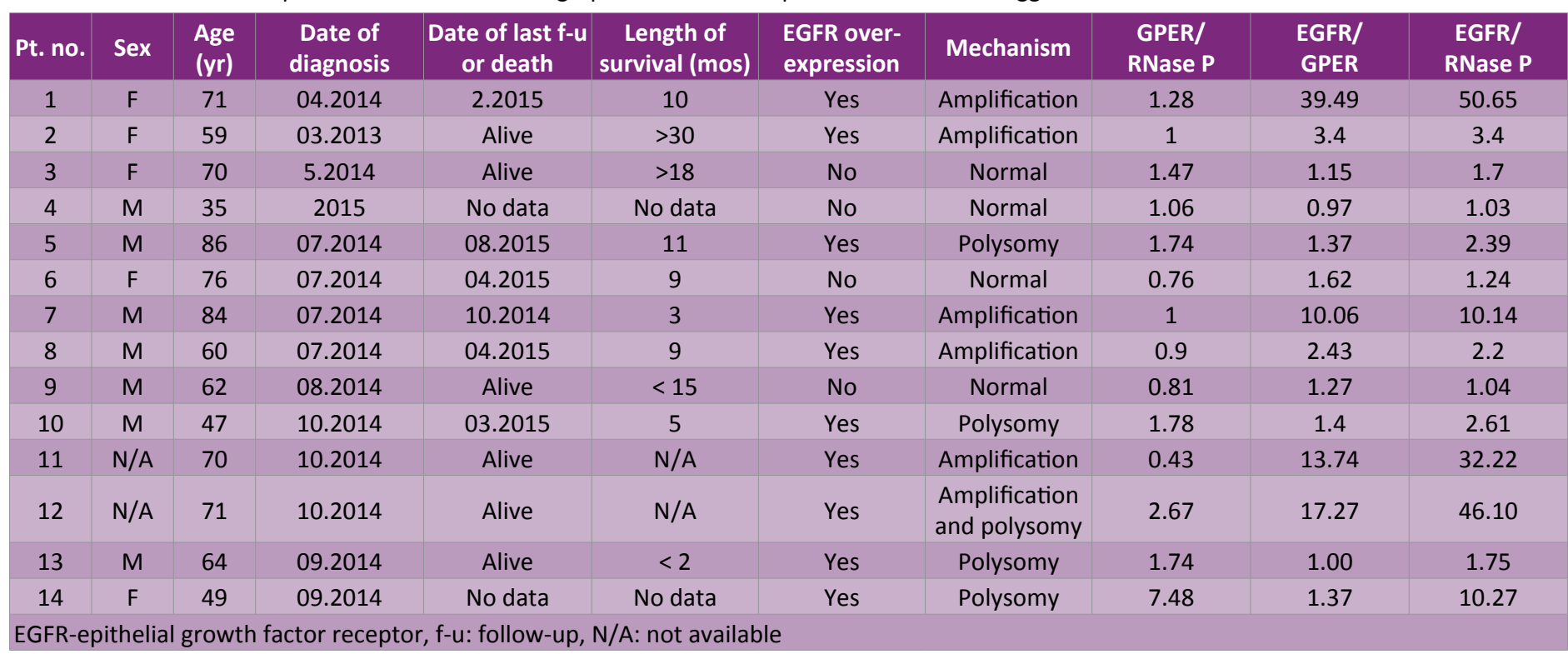


Demographics and EGFR expression status and suggested mechanism with the comparison to the clinical parameters and outcome are shown on Table 1.

\section{Immunostaining with GNP-conjugated anti- EGFR antibodies and comparison to routine pathologic staining}

EGFR-conjugated GNPs were detected following immunostaining of EGFR-overexpressing tumor samples. Hyperspectral imaging was performed on the tumor and surrounding neural tissue under different magnifications [20]. Representative results are presented in Figures 2. High reflectance at the peak GNPs wavelength ( $550 \mathrm{~nm}$ to $660 \mathrm{~nm}$ ) was found in areas identified histologically as GBM, and also in areas considered histologically to be free of tumor.

\section{Electron microscopy for direct visualization of GNPs in the tissue}

On scanning of the GNP-stained slides with a high-resolution scanning electron microscope $\left(\right.$ airSEM $^{\mathrm{TM}}$ ) [21], the anti-EGFRconjugated GNPs could be visualized in the tissue (Figure 3). As shown in Figure 3, a higher concentration was detected in cells in the tumor area than in the infiltrating cells in the adjacent area. Some areas did not stain. The GNP-stained slides showed infiltrating cells that were not detected on the H\&E-stained slides.

\section{Discussion}

On DNA analysis of the expression of the EGFR gene in fresh GMB samples from 14 patients, we found that $71 \%$ of the samples had increased levels of EGFR expression via either polysomy of chromosome 7 or amplification of the EGFR gene. Patients whose samples were characterized by an overexpression of EGFR died within 3-10 months, whereas 2 of the patients with normal EGFR expression were still alive 9 and $<15$ months after surgery. These findings suggest a possible prognostic value of EGFR overexpression in GBM. There is currently no known link

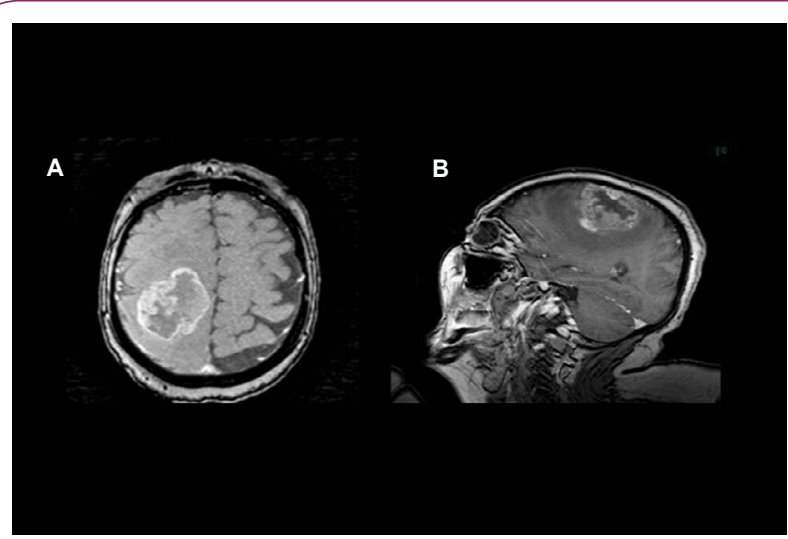

Figure 1 MRI brain imaging. T1 axial (A) and sagital (B) post contrast images showing a large intraxial heterogeneous enhancing mass with local infiltration and mass effect probably consistent with high grade tumor.

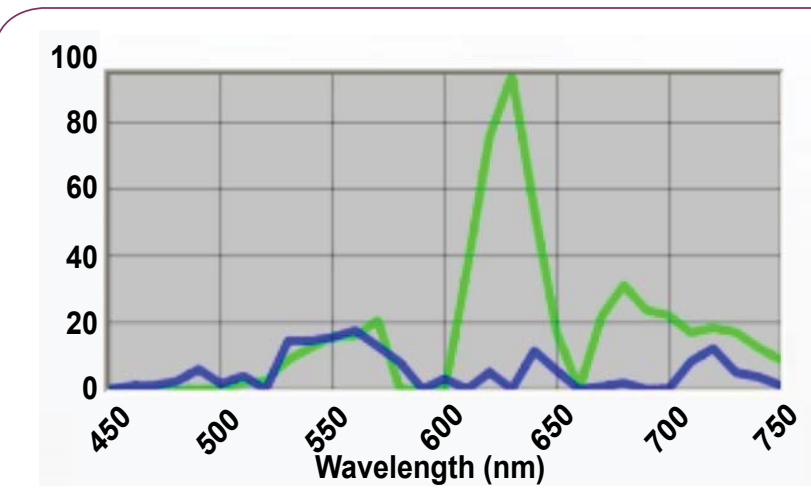

Figure 2 Hyperspectral spectra of tumor (green line) and surrounding neural tissue (blue line). The $Y$ axis is the reflectance intensity in arbitrary intensity units (IU).

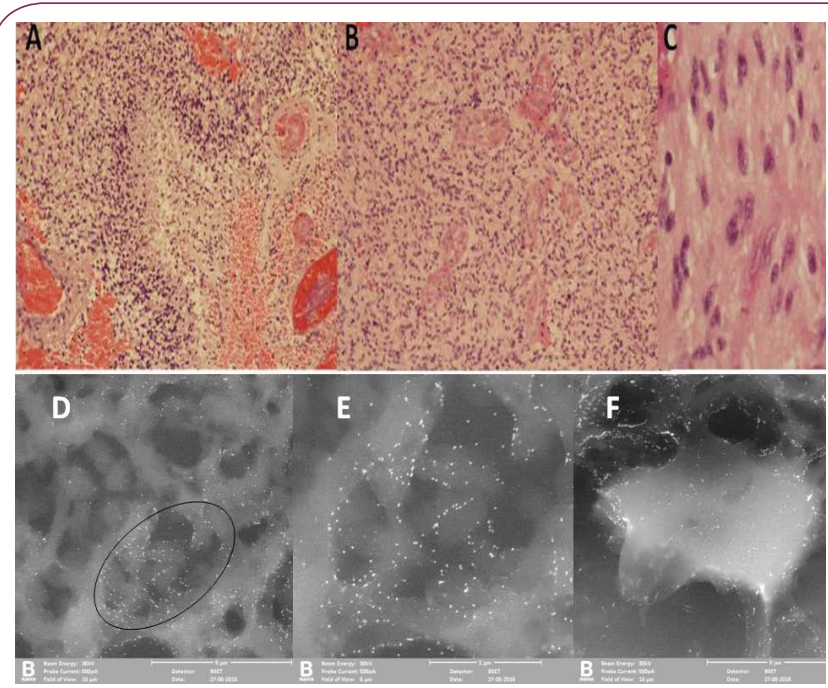

Figure 3 Histopathology of GBM and airSEM. Upper line: (A) pseudo palisading in necrotic area (X5), (B) microvascular proliferation (X10) and (C) mitosis (X20). Lower line: airSEM measurements of tumor and healthy regions of GBM in micrometric (D) and nanometric (E) resolutions. (D). Tumor (up to the dark line) and healthy (down to the dark line) sites of GBM tissue in brain. (E) and (F). airSEM pictures of healthy and tumor sites. GNPs appear as bright dots. Note: An evident difference in the GNPs concentration is clearly seen.

between chromosome 7 polysomy and GBM prognosis, and findings in the literature for the prognostic significance of EGFR overexpression are contradictory. One study suggested that it is indicative of poorer outcome [19] but others failed to support this conclusion $[5,17,18,22]$, except in pediatric GBM, in which overexpression of EGFR was linked to a worse prognosis [23]. A decreased expression of EGFR is clearly not associated with tumor recurrence [5].

Whether or not EGFR overexpression has predictive value for outcome, it apparently has importance as a marker of tumor borders and tumor-cell infiltration. On the basis of studies showing that monoclonal antibodies can be utilized to identify tumor borders [24], we immunostained samples of GBM with anti-EGFR antibodies conjugated to GNP. In this manner, we 
were able to differentiate the tumor tissue even from brain tissue considered healthy by standard histological methods. On both reflectance spectroscopy and electron microscopy, cells infiltrating surrounding areas could be clearly identified.

The high levels of EGFR present in GMB makes them a potentially valuable tool for targeted imaging and selective detection of tumor cells within normal brain parenchyma using monoclonal anti-EGFR antibodies conjugated to GNPs. Other studies have used anti-EGFR mutant antibody conjugated to iron oxide nanoparticles [25]. The iron oxide nanoparticles effectively reached the brain tumor by convection-enhanced delivery $[25,26]$ which bypasses the blood-brain barrier for more efficient tumor penetration [26].

Additionally, given the difficulty of complete resection of GBM tumors because of their diffuse structure and highly aggressive behavior, by using GNPs conjugated to anti-EGFR antibodies, researchers may potentially be able to preoperatively map the entire extent of tumor infiltration in order to guide the surgeon. GNP technology can also be used intraoperatively as a contrast agent on magnetic resonance imaging to highlight areas of unresected tissue that may not be visible with conventional intraoperative imaging [27].

Several groups have employed conjugated anti-EGFR antibodies in the treatment of cancer, either as a drug delivery vehicle [28] or as an aid to kill tumor cells by photothermal or radiofrequency ablation [29-32]. In one study, GNPs were used for radiofrequency ablation of pancreatic and colorectal adenocarcinomas in animal models [33]. Although the high levels of EGFR in some forms of GBM also make it an accurate and desirable therapeutic target, current therapies targeting EGFR, including tyrosine kinase inhibitors and RNA interference, have proved to be ineffective $[7,8,34]$. Therefore, attention is being addressed to other means: the delivery of novel antitumor agents across the blood-brain barrier, gene therapy, and brain tumor vaccines [35-37]. One study found that the use of metallic nanoparticles conjugated to anti-EGFR antibody combined with cisplatin could eradicate tumor cells when subjected to radiation in vitro [38]. It may therefore be possible to conjugate chemotherapeutic agents to GNP along with anti-EGFR antibodies to facilitate the accurate in vivo delivery of these agents to the tumor bed.

There are some drawbacks to conjugation of monoclonal antibodies to nanoparticles, particularly the large size of the conjugate and difficulty in achieving the necessary chemical reaction for conjugation [27]. Furthermore, the use of GNP in particular poses some risk. Although gold is inert, it has a tendency to aggregate in blood vessels, which may lead to toxicity and renal failure. Therefore, the GNP dosage must be carefully titrated with renal function [39].

GNP conjugated to EGFR may enable detection of tumor margins when EGFR is overexpressed. This may limit the use of this technique to approximately $60 \%$ of the tumors, in comparison to the existing 5 ALA methods, which has a similar $40 \%$ suboptimal detection ability [40]. Therefore, the GNP-EGFR new method suggested herein may be as good as 5 ALA, if not better. When comparing coloring methods, two techniques have been investigated in vivo to date. The nano-encapsulated visible dye was tested in animal model (rats) for intraoperative visual delineation of tumor margins [41] and the intravenous fluorescein sodium detected by specific camera [42]. Liposomal nanocarriers containing Evans blue dye (nano-EB) clearly aids in distinguishing tumor tissue from healthy tissue in rat model. The injection of unencapsulated EB failed to distinguish tumor from healthy brain tissue due to diffusion of dye from the tumor into healthy tissue [41]. Using high doses of fluorescein sodium under white light somewhat improved total resection of GBM. The new fluorescein-specific camera that allows for high-quality intraoperative imaging while using very low dose fluorescein has improved this method. However, fluorescein sodium does not appear to selectively accumulate in astrocytoma cells but in extracellular tumor cell-rich locations. It shows leakage areas of compromised blood-brain barrier, in correlation with enhanced areas on magnetic resonance imaging [42].

There are some limitations to this study. We describe a small cohort that cannot be analyzed statistically. Although this study has shown that GNPs conjugate with anti-EGFR antibodies preferentially home to the GBM tissue expressing EGFR, this concept still needs to be tested in vivo. As not all tumors show EGFR overexpression, and healthy neuronal tissue may also express this receptor, the efficiency of the method is yet to be examined. The added value of detecting GNPs intraoperatively depends on the structures and resectability of the tumor. This study has not yet intervened with GNP injection to patients, and therefore no measurement of toxicity is available. It is possible that in an in vivo system the conjugated GNPs could behave differently than in an in vitro system. To date, the use of GNP did not reveal any toxicity. We have set the foundation for a possibly useful tool to help achieve full resection of GBM. The next phase of this work will be to test this methodology in vivo. This study could be useful for future pre- and peri-operative mapping of tumor margins and for delivery of direct chemotherapy to the tumor cells. Although 5 ALA has been investigated for the last decade, and proven to have a good safety profile $[40,43,44]$ with relative efficacy $[43,44]$, the benefits of its use are less than expected. The differentiation between tumor and normal cerebral tissue was suboptimal. Herein, we suggest a new method, that may overcome some of the obstacles. However, further investigation is needed.

\section{Conclusions}

In summary, we have shown that GBM overexpresses EGFR mechanisms by polysomy of chromosome 7 and amplification. In addition, we show that GNPs conjugated to anti-EGFR antibody preferentially home to areas of tumor within normal brain parenchyma and are therefore useful for the detection of tumor cells that infiltrate surrounding neuronal tissue. They may also serve as a tool for preoperative and intraoperative mapping of resection margins and possibly for the delivery of chemotherapeutic agents directly to the tumor bed and in vivo killing of tumor cells using focused radiation. 


\section{References}

1 Layfield LJ, Willmore C, Tripp S, Jones C, Jensen RL (2006) Epiderma growth factor receptor gene amplification and protein expression in glioblastoma multiforme: Prognostic significance and relationship to other prognostic factors. Appl Immunohistochem Mol Morphol 14: 91-96.

2 Cancer Genome Atlas Research Network (2008) Comprehensive genomic characterization defines human glioblastoma genes and core pathways. Nature 455: 1061-1068.

3 Mizoguchi M, Betensky RA, Batchelor TT, Bernay DC, Louis DN, et al. (2006) Activation of STAT3, MAPK, and AKT in malignant astrocytic gliomas: Correlation with EGFR status, tumor grade, and survival. $J$ Neuropathol Exp Neurol 65: 1181-1188.

4 Lopez-Gines C, Gil-Benso R, Ferrer-Luna R, Benito R, Serna E, et al. (2010) New pattern of EGFR amplification in glioblastoma and the relationship of gene copy number with gene expression profile. Mod Pathol 23: 856-865.

5 Shinojima N, Tada K, Shiraishi S, Kamiryo T, Kochi M, et al. (2003) Prognostic value of epidermal growth factor receptor in patients with gioblastoma multiforme. Cancer Res 63: 6962-6970.

6 Bieńkowski M, Piaskowski S, Stoczyńska-Fidelus E, Szybka M, Banaszczyk M, et al. (2013) Screening for EGFR amplifications with a novel method and their significance for the outcome of glioblastoma patients. PLoS One 8: e65444.

7 Gan HK, Burgess AW, Clayton AHA, Scott AM (2012) Targeting of a conformationally exposed, tumor-specific epitope of EGFR as a strategy for cancer therapy. Cancer Res 72: 2924-2930.

8 Sun Y, Zhang W, Chen D, Lv Y, Zheng J, et al. (2014) A glioma classification scheme based on co-expression modules of EGFR and PDGFRA. Proc Natl Acad Sci USA 111: 3538- 3543.

9 Gorlia T, van den Bent MJ, Hegi ME, Mirimanoff RO, Weller M, et al. (2008) Nomograms for predicting survival of patients with newly diagnosed glioblastoma: Prognostic factor analysis of EORTC and NCIC trial 26981-22981/CE.3. Lancet Oncol 9: 29-38.

10 Stewart LA (2002) Chemotherapy in adult high-grade glioma: a systematic review and meta-analysis of individual patient data from 12 randomised trials. Lancet 359: 1011-1018.

11 Stupp R, Hegi ME, Mason WP, van den Bent MJ, Taphoorn MJ, et al. (2009) Effects of radiotherapy with concomitant and adjuvant temozolomide versus radiotherapy alone on survival in glioblastoma in a randomised phase III study: 5-year analysis of the EORTC-NCIC trial. Lancet Oncol 10: 459-466.

12 Choi JS, Jun YW, Yeon SI, Kim HC, Shin JS, et al. (2006) Biocompatible heterostructured nanoparticles for multimodal biological detection. J Am Chem Soc 128: 15982-15983.

13 Ankri R, Peretz V, Motiei M, Popovtzer R, Fixler D (2012) A new method for cancer detection based on diffusion reflection measurements of targeted gold nanorods. Int J Nanomedicine 7: 449-455.

14 Ankri R, Duadi H, Motiei M, Fixler D (2012) In vivo tumor detection using diffusion reflection measurements of targeted gold nanorods A quantitative study. J Biophotonics 5: 263-273.

15 Fixler D, Ankri R (2013) Subcutaneous gold nanorods (corrected) detection with diffusion reflection measurement. J Biomed Opt 18 61226 .
16 Louis DN, Ohgaki H, Wiestler OD, Cavenee WK, Burger PC, et al. (2007) The 2007 WHO classification of tumours of the central nervous system. Acta Neuropathol 114: 97-109.

17 Ankri R, Melzer S, Tarnok A, Fixler D (2015) Detection of gold nanorods uptake by macrophages using scattering analyses combined with diffusion reflection measurements as a potential tool for in vivo atherosclerosis tracking. Int J Nanomedicine 10: 4437-4446.

18 Melzer S, Ankri R, Fixler D, Tarnok A (2015) Nanoparticle uptake by macrophages in vulnerable plaques for atherosclerosis diagnosis. J Biophotonics 8: 871-883.

19 Ai H, Fang M, Jones SA, Lvov YM (2002) Electrostatic layer-bylayer nanoassembly on biological microtemplates: platelets. Biomacromolecules 3: 560-564.

20 Ankri R, Taitelbaum H, Fixler D (2011)Phantom experiments of the photon migration model in tissues. Open Opt J 5 Suppl 1-M4: 28-32.

21 Ankri R, Ashkenazy A, Milstein Y, Brami Y, Olshinka A, et al. (2016) Gold nanorods based air scanning electron microscopy and diffusion reflection imaging for mapping tumor margins in squamous cell carcinoma cell. ACS Nano 10: 2349-2356.

22 Pfaffl MW, Horgan GW, Dempfle L (2002) Relative expression software tool (REST) for group-wise comparison and statistical analysis of relative expression results in real-time PCR. Nucleic Acids Res 30: e36.

23 Simmons ML, Lamborn KR, Takahashi M, Chen P, Israel MA, et al. (2001) Analysis of complex relationships between age, p53, epidermal growth factor receptor, and survival in glioblastoma patients. Cancer Res 61: 1122-1128.

24 Mitchell DA, Fecci PE, Sampson JH (2008) Immunotherapy of malignant brain tumors. Immunol Rev 222: 70-100.

25 Hadjipanayis CG, Machaidze R, Kaluzova M, Wang L, Schuette AJ, et al. (2010) EGFRvIII antibody-conjugated iron oxide nanoparticles for magnetic resonance imaging-guided convection-enhanced delivery and targeted therapy of glioblastoma. Cancer Res 70: 6303-6312.

26 Raghavan R, Brady ML, Rodríguez-Ponce MI, Hartlep A, Pedain C, et al. (2006) Convection-enhanced delivery of therapeutics for brain disease, and its optimization. Neurosurg Focus 20: E12.

27 Yu MK, Park J, Jon S (2012) Targeting strategies for multifunctional nanoparticles in cancer imaging and therapy. Theranostics 2: 3-44.

28 Park C, Youn H, Kim H, Noh T, Kook YH, et al. (2009) Cyclodextrincovered gold nanoparticles for targeted delivery of an anti-cancer drug. J Mater Chem 19: 2310-2315.

29 El-Sayed IH, Huang X, El-Sayed MA (2006) Selective laser photothermal therapy of epithelial carcinoma using anti-EGFR antibody conjugated gold nanoparticles. Cancer Lett 239: 129-135.

30 Jakobsohn K, Motiei M, Sinvani M, Popovtzer R (2012) Towards realtime detection of tumor margins using photothermal imaging of immune-targeted gold nanoparticles. Int J Nanomedicine 7: 47074713.

31 Melancon MP, Lu W, Yang Z, Zhang R, Cheng Z, et al. (2008) In vitro and in vivo targeting of hollow gold nanoshells directed at epidermal growth factor receptor for photothermal ablation therapy. Mol Cancer Ther 7: 1730-1739.

32 Melancon MP, Lu W, Zhong M, Zhou M, Liang G, et al. (2011) Targeted multifunctional gold-based nanoshells for magnetic resonanceguided laser ablation of head and neck cancer. Biomaterials 32: 7600-7608. 
33 Löw K, Wacker M, Wagner S, Langer K, von Briesen H (2011) Targeted human serum albumin nanoparticles for specific uptake in EGFRExpressing colon carcinoma cells. Nanomedicine 7: 454-463.

34 Padfield E, Ellis HP, Kurian KM (2015) Current therapeutic advances targeting EGFR and EGFRvIII in glioblastoma. Front Oncol 5: 5.

35 Chen Y, Liu L (2012) Modern methods for delivery of drugs across the blood-brain barrier. Adv Drug Deliv Rev 64: 640-665.

36 Gustafson MP, Knutson KL, Dietz AB (2008) Therapeutic vaccines for malignant brain tumors. Biologics 2: 753-761.

37 Murphy AM, Rabkin SD (2013)Current status of gene therapy for brain tumors. Transl Res 161: 339-354.

38 Setua S, Ouberai M, Piccirillo SG, Watts C, Welland M (2014) Cisplatintethered gold nanospheres for multimodal chemo-radiotherapy of glioblastoma. Nanoscale 6: 10865-10873.

39 Doudi M, Setorki M (2014) The effect of gold nanoparticle on renal function in rats. Nanomedicine J 1: 171-179.

40 Teixidor P, Arráez MÁ, Villalba G, Garcia R, Tardáguila M, et al. (2016)
Safety and efficacy of 5-aminolevulinic acid for high grade glioma in usual clinical practice: a prospective cohort study. PLoS One 11: e0149244.

41 Roller BT, Munson JM, Brahma B, Santangelo PJ, Pai SB, et al. (2015) Evans blue nanocarriers visually demarcate margins of invasive gliomas. Drug Deliv Transl Res 5: 116-124.

42 Diaz RJO, Dios RRE, Hattab EM, Burrell K, Rakopoulos P, et al. (2015) Study of the biodistribution of fluorescein in glioma-infiltrated mouse brain and histopathological correlation of intraoperative findings in high-grade gliomas resected under fluorescein fluorescence guidance. J Neurosurg 122: 1360-1369.

43 Stummer W, Pichlmeier U, Meinel T, Wiestler OD, Zanella F, et al. (2006) ALA-Glioma study group. Fluorescence-guided surgery with 5-aminolevulinic acid for resection of malignant glioma: A randomised controlled multicentre phase III trial. Lancet Oncol 7: 392-401.

44 Widhalm G (2014) Intra-operative visualization of brain tumors with 5-aminolevulinic acid-induced fluorescence. Clin Neuropathol 33: 260-278. 